\title{
Comparison of Some Somatic and Reproductive Characters for Tow Brood Stock of Cyprinus Carpio $L$.
}

\author{
Muthana Sabah Azawy \\ Ammar Abid-Alwahid Issa \\ Technical College / Al-Mussaib/ Al-Furat Al-Awsat Technical University-51009 \\ M.Sabah41@yahoo.com
}

\begin{tabular}{l} 
ARTICLE INFO \\
\hline Submission date: $16 / 9 / 2018$ \\
Acceptance date: $7 / 10 / 2018$ \\
Publication date: $10 / 3 / 2019$ \\
\hline
\end{tabular}

\begin{abstract}
Studies have been done in seven hatchenis in in Mahawil district/Babil from the period 15/3 to 15/5 to identify the body and reproductive characteristics between common carp brood stock which was imported in 2009 containing this shiny line of scales that exists dorsal fin of carp and which is without shiny line of scales was imported in 1982. Body characteristic results showed significant difference $P<0.05$ between two brood stock according to body depth/standard length feature, so they reached an average of this feature in common carp shiny line of scales $0.003 \pm 0.389$ higher than those containing no shiny line reached $0.002 \pm 0.369$, whereas no significant differences was shown between two brood stock for fish weight and head length/ standard length. Reproductive characteristic study showed significant difference $\mathrm{P}$ $<0.05$ for shiny line brood stock for each total eggs weight(gm), eggs number /gm eggs, eggs weight $/ \mathrm{kg}$ fish and fertilization percentage characteristic, which reached an average of $26.62 \pm 520.28,4.46 \pm$ $764.60,94.76 \pm 159.2,0.33 \pm 82.91$ respectively, higher than of those without shiny line brood stock which reached average of $21.32 \pm 461.42,4.44 \pm 711.15,4.42 \pm 147.73$ respectively, whereas significant difference was shown in egg diameter characteristic for containing no shiny line brood stock which reached $0.01 \pm 1.411$ where brood stock of shiny line reached average of $0.01 \pm 1.38$ and no significant differences were showed between two brood stock in eggs number/ $\mathrm{kg}$ fish. That an increase of crossbreeding between different lines for common carp of brood stock do in improve somatic and reproductive characteristics of inbreeding and prevents deteriorating.
\end{abstract}

Key Words: Cyprinus carpio, Standard length, Eggs weight, Fertilization percentage.

(C) Journal of University of Babylon for Pure and Applied Sciences (JUBES) by University of Babylon is licensed under a $\underline{\text { Creative }}$ Commons Attribution 4. 0 International License 


\section{مقارنة بهذ الصفات الجسمية والتكاثرية لأُمهات قطيهين من الكارب \\ Cyprinus carpio L. الاعتيادخ

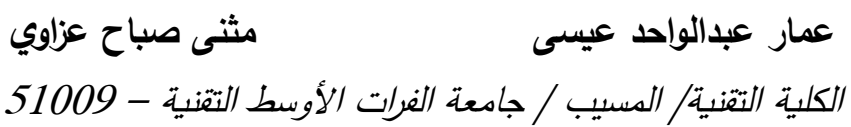

الخلاصة

أجريت الدراسة في سبع مفاقس في قضاء المحاويل / محافظة بابل للمدة من 2018/3/15 لغاية 2018/5/15، من أجل الجية

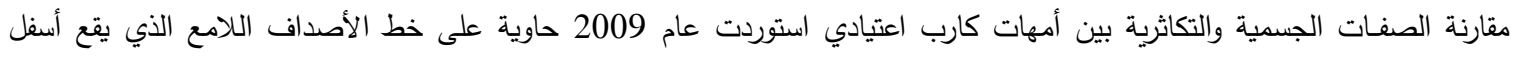

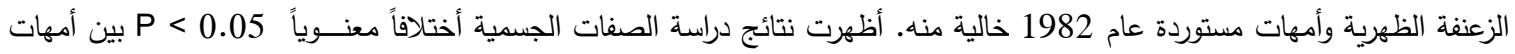

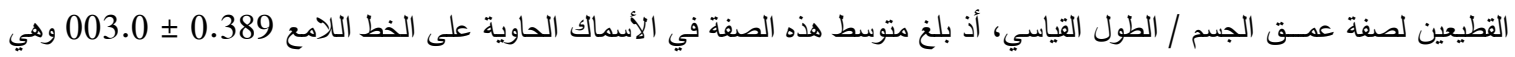

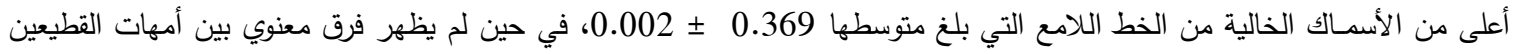
لوزن السمـكة وطول الرأس / الطول القياسي.أما نتائج دراسة الصفات التكاثرية أظهرت تفوقاً معنوياً

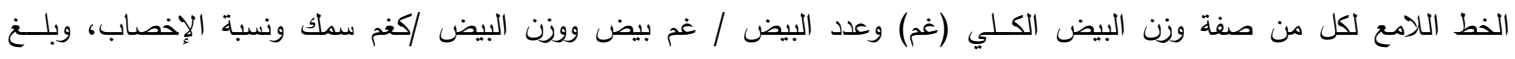

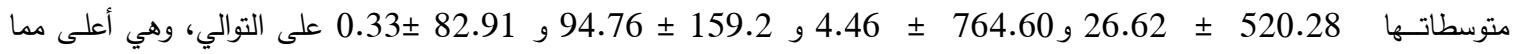

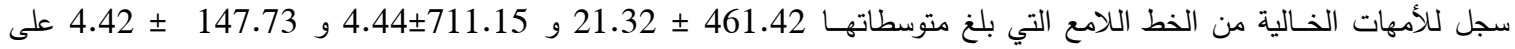

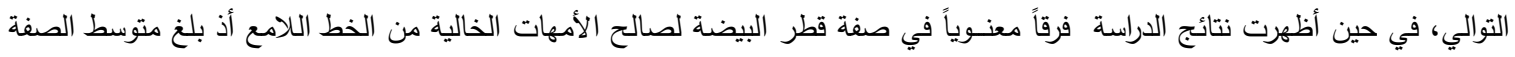

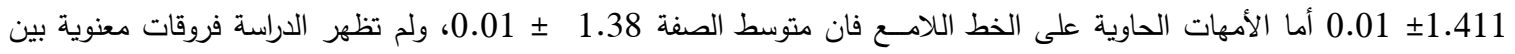
القطيعين في صفة (عدد البيض/ كغ سمك). أن أجراء التضـريب بين خطوط مختلفة لأُمهات أسماك الكارب الاعتيادي تعمل على تحسين الصفات الجسمية والتكاثرية من التربية الداخلية وتمنع تدهورها.

الكلمات المفتاحية :- الكارب الاعتيادي، الطول القياسي، وزن البيض، نسبة الإخصاب

\section{المقدمة}

يمثل قطاع الثروة السمكية احد القطاعات الواعدة في الجانب الاقتصادي وذات مردود مهم لدول العالم لما لها من دور في تحقيق درجـات عاليه من الاكتفاء الذاتي [1]، وتعد الأسماك من المصادر الرئيسة للبروتين كونها تستطيع أن توفر جـزءاً كبيراً من الغـــاء ولا سيما في الــدول النامـية [2]، كون النبات يحتوي على كميات قليلة من المحتوى ألبروتيني الذي لا يسد متطلبات جسم الأنسان منها [3]. تعد الأسماك مصدراً رئيساً لغذاء الإنسان

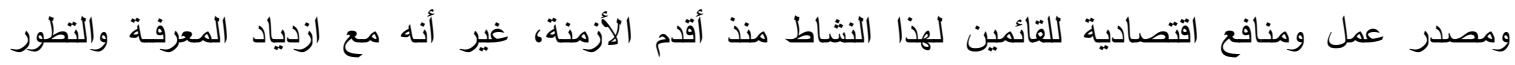
الديناميكي لمصايد الأسماك تبين أن موارد الأحياء المائية رغم تجددها فأنها تحتاج أن تدار بثكل رشيد أذا أنا أردنا أن نساهم على الدوام في تحسين الأوضاع الغذائية الاقتصادية لسكان هذا العالم الذي يتزايد أعدادهم باطراد[4]. مما دفعـت الإنسان إلى الاهتمـام في تطوير واستغلال تربية الأسماك التي تمتاز بمقاومة عالية للظروف المختلفة وسرعة نمـو جيدة فتأتي أسمـاك الكارب في مقدمة تلك الأسماك [5]. يمتلك العراق العديد من المسطحات المائية الصالحة لتربية الأسماك وأكثارها تكفي الحاجة المحلية أو تزيد في حالة استغلالها بشكل أفضـل كنهري دجلة 
والفرات والفروع والروافد المرتبطة بها وشط العرب والخزانات والبحيرات والاهوار والتي تثكل بمجموعها مايقارب 5\% من مساحة العراق الكلية [6 ; 7]، فضلاً عن وجود شبكة بزل واسعة ترتبط بالمصب العام تمتد من شمال بغداد وحتى الخليج العربي [8]، إضافة إلى الأحواض الاصطناعية التي تتنشر بمناطق واسعة في وسط وجنوب العراق التي تزايدت إعدادها في السنوات الأخيرة لتربية الأسماك بشكل ملحوظ. وبسبب عدم وجود برامج تربية وراثية مختلفة أثر سلباً في الصفات المظهرية والصفات الكمية مما أدى إلى ظهور صفات غير مرغوب بهاب للقهات للقعان المربات محلياً مثل قلة النمو وقلة كفاءة التحويل الغذائي وتطاول الجسم وقلة مقاومة الأمـراض بالأضافة إلى تـدهور الصفات الوراثية [9]. لذا أصبحت أسماك الكارب الاعتيادي محور العديد من الدراسات في العالم لأنها من أهم عوائل أسمـاك المياه العذبة، تتاولت هذه الدراسات كل ما يتعلق بدراسة التطور المظهري والوراثي والتركيب الوراثي وتأثير التدجين للأسماك [ 10و 11 ]. عملياً لم تدخل أمهات جديدة إلى المزارع السمكية منذ عام 1982 مما أدى إلى تدهور اداء الأسماك القديمة بسبب استمرار التربية الداخلية لقطعان الأمهات، لذا قامت وزارة الزراعة عام 2000 بأدخال أسماك جديدة ولكن لم تتتشر تراكيبها الوراثية بسبب التحفظ عليها نتيجة أصابتها بالأمراض ومن ثمرات اختفت في أحداث 2003، وفي عام 2009 تم استيراد أسماك جديدة وزعت إلى أغلب المفاقيس في بابـل لأجراء التضريب بينها وبين الأسماك المستوردة 1982 [12]. بعد ثماني سنوات من التريبة وظهور أجيال جديدة سواء الأمهات المستوردة عام 2009 أو القــديمة أو الناتجة من التضريب قد يكون هناك اختلاف مابين هذه المجاميع من لنابن حيث أنتاجها التكاثري لذا تهدف الدراسة إلى ربط الصـفات الجسمـية للأمهات مع قيم أنتاجها التكاثري.

المواد وطرائق العمل اختيار مواقع الدراسة

أجريت الدراسة في سبعة مفاقس لتكثير أسماك الكارب الاعتيادي في قضاء المحاويل الذي يبعد عن محافظة بابل

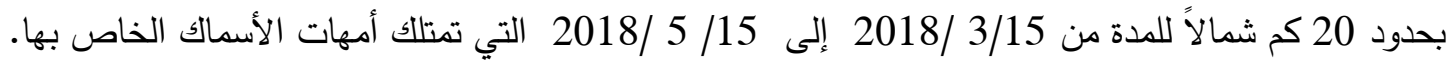
قطيع الأمهات

تم العمل على قطعان أمهات أسماك الكارب الاعتيادي المعدة للتكثير في سبع مفاقس، مجموعها الكلي (301) منها الجيل القديم الذي أدخل عام 1982 التي يعتقد أن صفاتها الجسمية وإنتاجها التكاثري تأثر من التربية الداخلية وكان عـدها (198) صورة (1)، وعدد من أمهات الجيل الجديد التي أدخلت عام 2009 وكان عددها (103) صورة (2) التي نستطيع أن نميزها من خلال وجود خط الأصداف اللامع أسفل الزعنفة الظهرية، مقسمة إلى 14 وجبة تكثير وأجريت المقارنة بينهما من خلاله دراسة. أولاً: وجود أو عدم وجود خط الأصداف اللامع. ثانياً : قياس الصفات الجسمية وبحسب الآتي : وجدود :

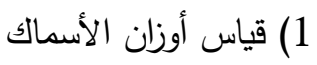
وزنت الأناث المختارة بوساطة ميزان. وبعدها يتم تعليمها بعلامات حسب أوزانها. 2) قياس أطوال الأسماك وكما يأتي : أ) الطول الكلي

تم قياس أطوال جميع الأسماك ابتدأ من مقدمة الرأس (بداية المخطم Snot) وحتى نهاية الزعنفة الذنبية [13]. 
ب) الطول القياسي قيس طول كل سكة ابتداء من مقدمة الرأس (بداية المخطم) وحتى قاعدة الزعنفة الذنبية [ 14]. ج) طول الرأس قيست المسافة مابين مقدمة الرأس وحتى نهاية الغطاء الغلصمي. د) ارتفاع الجسم (عمق الجسم)

تم قياس أكبر عمق للسمكة والتي عادة ما تكون الجسافة العمودية مابين الزعنفة الظهرية وبداية الزعنفة الحوضية.

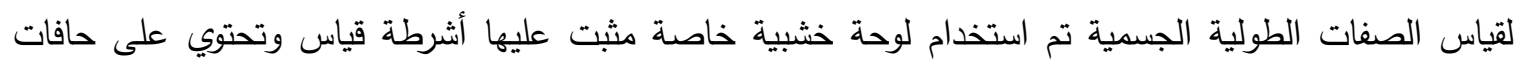

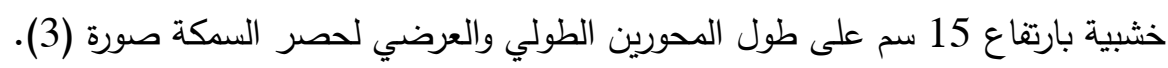

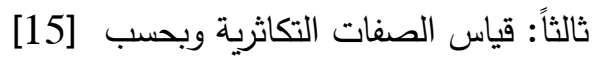
تتم بعد أجراء عملية التكثير الاصطناعي للأمهات وتثمل مايلي : 1- وزن البيض المستخرج من كل سمكة بوساطة ميزان.

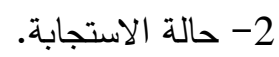

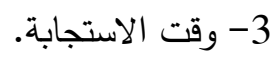
4- أخذ 1غم من البيض وحُفظ في أنبوبة اختبار حاوية على مادة الفورمالين.

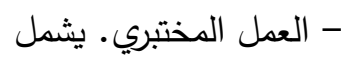

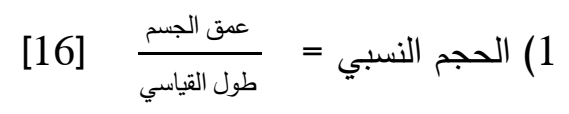
2) حساب طول الرأس/ الطول القياسي

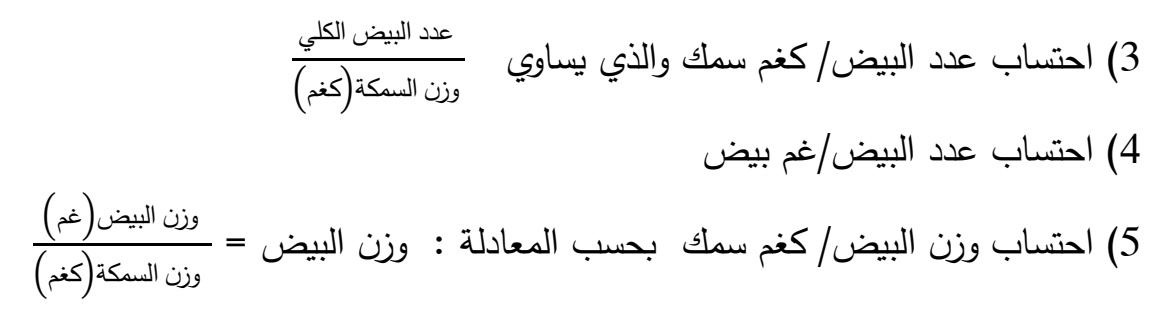
6) قطر البيضة (ملم) أخذت 10 بيوض مغردة من البيوض العائدة لكل سمكة خاضعة للدراسة وقيست أقطار تلك البيوض بوساطة عدسة (PEAK scale Lope 7x) والتي تكون قياساتها لأقرب 0.1 ملم ثم يؤخذ معدل القراءات. 7) نسبة الإخصاب (1x)

حُسبت نسبة الإخصاب بعد 12 ساعة من الإخصاب وذللك عن طريق وضع أنبوب بلاستك شفاف داخل

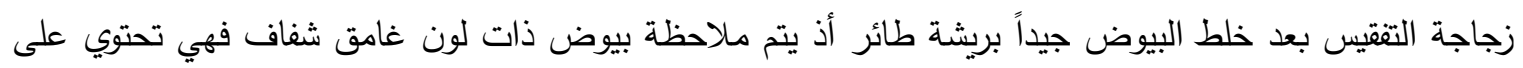

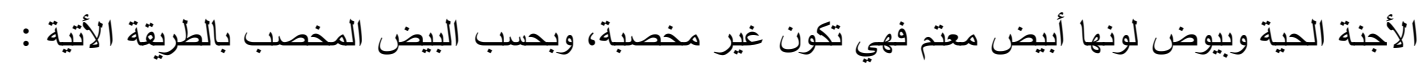

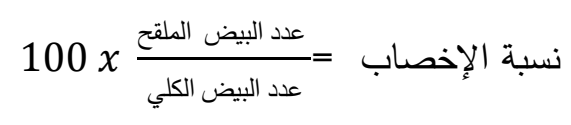




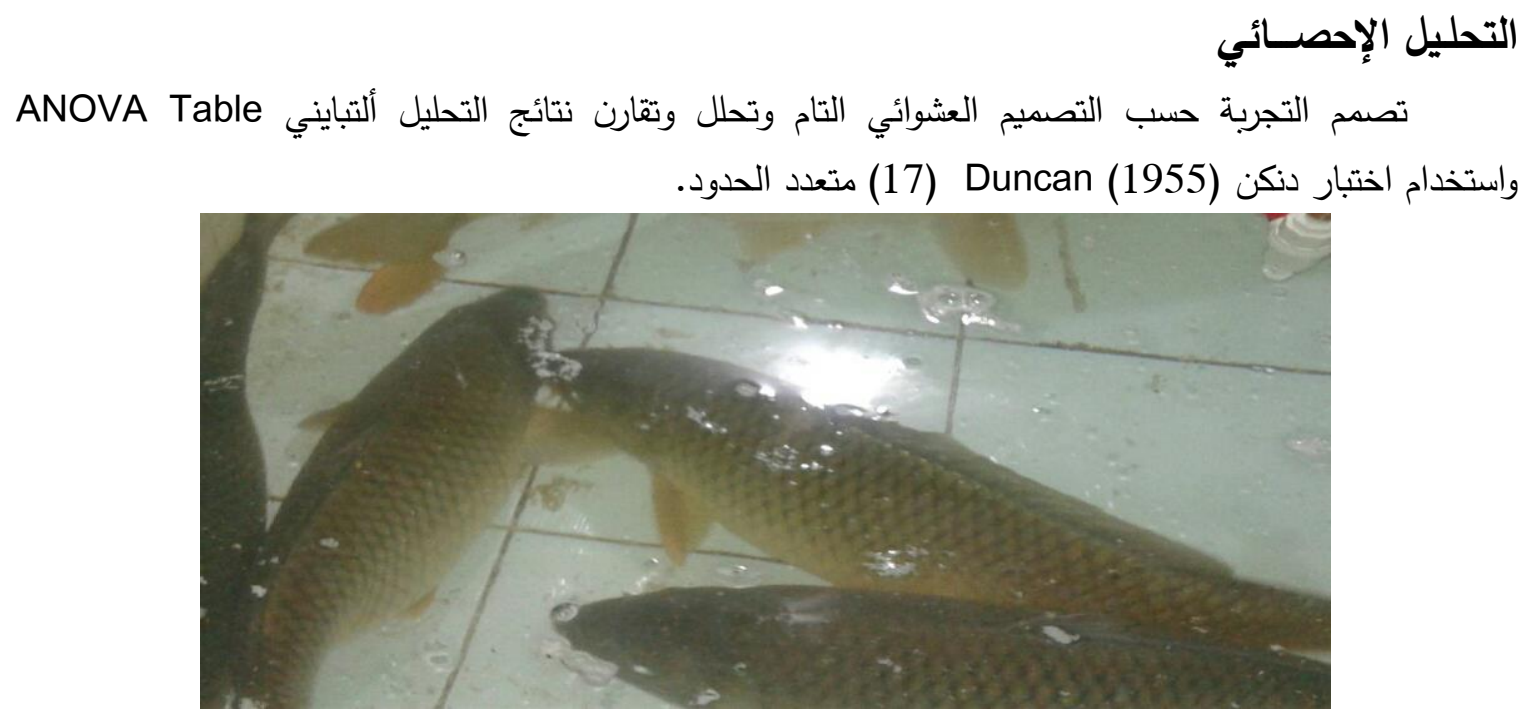

صورة رقم(1) يين عدم وجود خط الأصداف اللامع في جهة الظهرية لأمهات الكارب الاعتيادي Cyprinus .Carpio

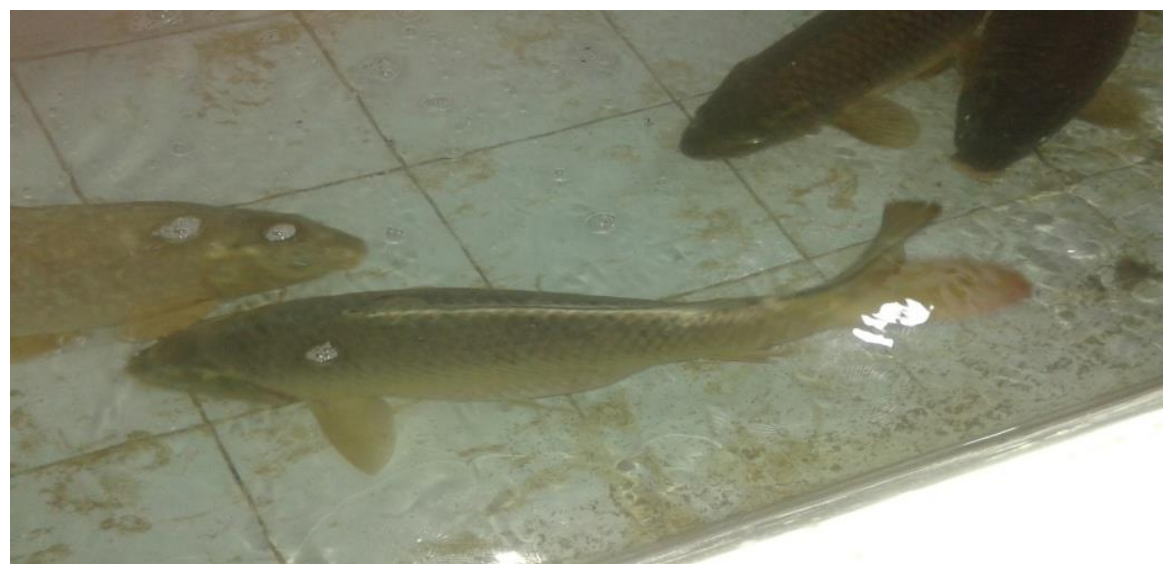

صورة رقم (2) يين موقع خط الأصداف اللامع في الجهة الظهرية لأمهات أسماك الكارب الاعتيادي .Cyprinus carpio

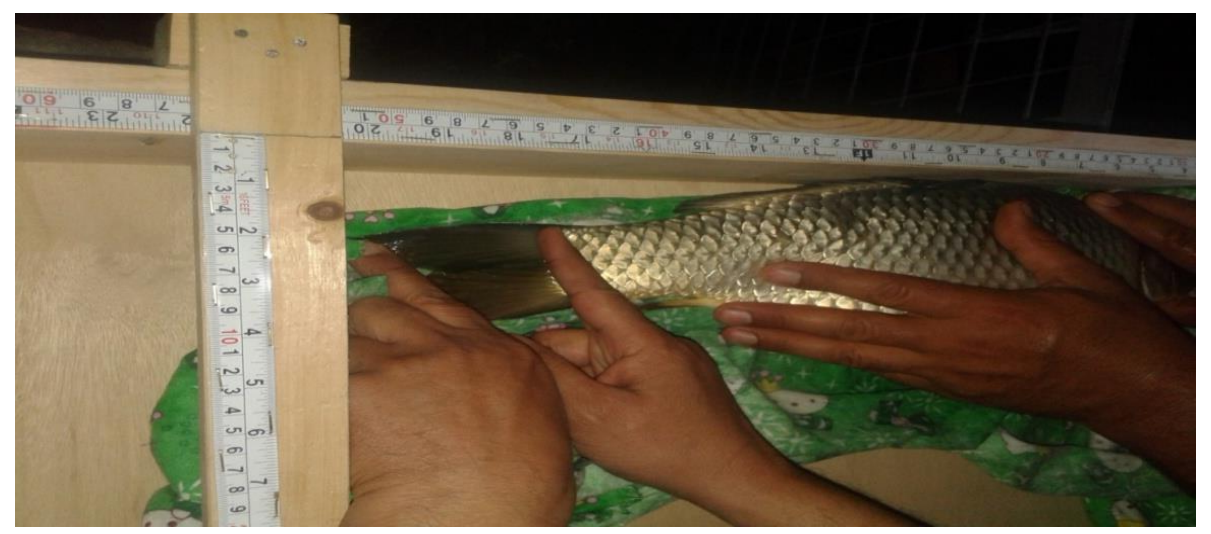


صورة رقم (3) يوضح استخدام ألوحة الخثبية المثبت عليها أشرطة قياس الصفات الطولية الجسمية النتائجج

أظهرت نتائج جدول (1) أن هناك فرقاً معنوياً 0.05 > P بين أمهات القطيعين لصفة عمق الجسم/

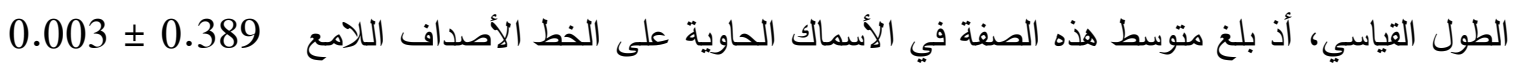

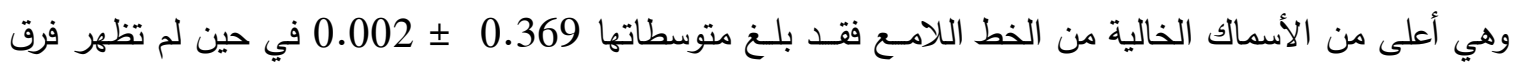

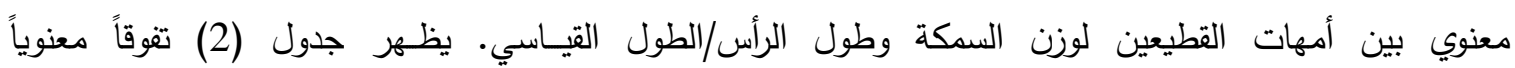

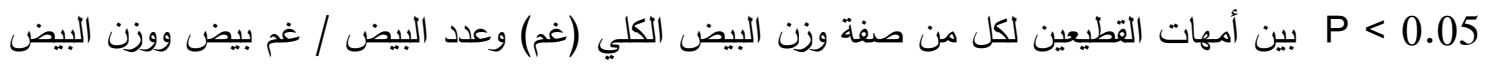
/كغم سمك ونسبة الإخصاب، أذ بلغ متوسطاتها في الأمهات الحاوية على الخط اللامع 520.28 ـ 52.62 ـ 26.62 و 764.60 × 4.46 و 159.2 × 94.76 و 82.91 ـ0.33 على التوالي. وهي أعلى من الأمهات الخاليـة

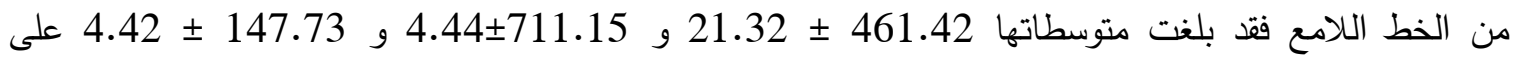
التوالي، في حين ظهر فرق معنوي في صفة قطر البيضة لصالح القطيع الخالي من الخط اللامع أذ بلغ متوسط

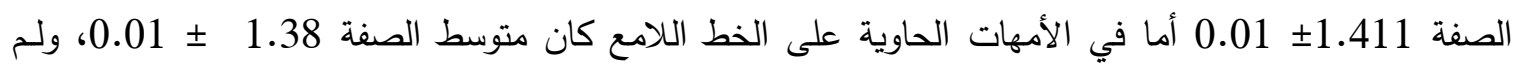

$$
\text { تظهر فروقات معنوية للقطيعين في صفة (عدد البيض/كغم سكك). }
$$

الجدول 1. المقارنة بين الأسماك الخالية من الخط اللامع وأسماك تحوي الخط الخط اللامع في كل من وزن السمكة

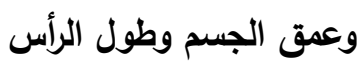

\begin{tabular}{|c|c|c|c|c|}
\hline \multicolumn{3}{|c|}{ الدتوسط × الخطأ القياسي } & \multirow[t]{2}{*}{ 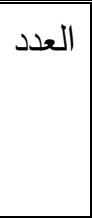 } & \multirow{2}{*}{ اللامع } \\
\hline 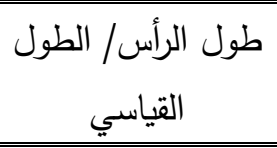 & عمق الجسم/ الطول & معدل وزن السمكة & & \\
\hline $\begin{array}{c}0.002 \pm 0.237 \\
a\end{array}$ & $\begin{array}{c}0.002 \pm 0.369 \\
b\end{array}$ & $\begin{array}{c}0.11 \pm 3.18 \\
a\end{array}$ & 198 & الخط اللامع خالية من \\
\hline $\begin{array}{c}0.002 \pm 0.234 \\
a\end{array}$ & $\begin{array}{c}0.003 \pm 0.389 \\
a\end{array}$ & $\begin{array}{c}0.15 \pm 3.29 \\
a\end{array}$ & 103 & الخط اللاكع تحوي على \\
\hline NS & * & NS & --- & مستوى المعنوية \\
\hline \multicolumn{5}{|c|}{ * (NS (P<0.05)، غير معنوي } \\
\hline
\end{tabular}


Journal of University of Babylon, Pure and Applied Sciences, Vol. (27), No. (1): 2019

الجدول 2. المقارنة بين الأسماك الخالية من الخط اللامع وأسماك تحوي الخط اللامع في صفات البيض

\begin{tabular}{|c|c|c|c|c|c|c|c|}
\hline \multicolumn{7}{|c|}{ المتوسط 土 الخطأ القياسي } & \multirow{2}{*}{ صفة وجود } \\
\hline قطر البيضة & الإخصابة & كغم سمك البيض/ & عدم البيض| & كغد البيض/ & وزن البيض & السمكة (كغد) وزن & \\
\hline \pm 1.411 & \pm 78.79 & \pm 147.73 & \pm 711.15 & \pm 280.72 & \pm 461.42 & \pm 3.18 & اسماك خالية الية \\
\hline 0.01 & 0.33 & 4.42 & 4.44 & 11.54 & 21.32 & 0.11 & من الخط اللامع \\
\hline a & b & $b$ & $b$ & a & $b$ & a & \\
\hline \pm 1.38 & \pm 82.91 & \pm 159.21 & \pm 764.60 & \pm 281.64 & \pm 520.28 & \pm 3.29 & اسماك تحوي \\
\hline 0.01 & 0.33 & 4.76 & 4.46 & 14.95 & 26.62 & 0.15 & على الخط \\
\hline $\mathrm{b}$ & a & a & a & a & a & a & الملامع \\
\hline$*$ & $*$ & * & * & NS & * & NS & مستوى المعنوية \\
\hline & & معنو & ، & ر معنوي & " & & \\
\hline
\end{tabular}

\section{المناقثة}

أظهرت نتائج الدراسة الحالية تفوق الأسماك التي تحوي صفات وراثية للأسماك المستوردة عام 2009

والحاوية على خط الأصداف اللامع على الأسماك التي استوردت عام 1982 الخالية من خط الأصداف اللامع في صفة عمق الجسم/الطول القياسي خلال في مدة التكثير • وهذا قد يكون ناتج من عملية التضريب بين الأجيال بعد التد

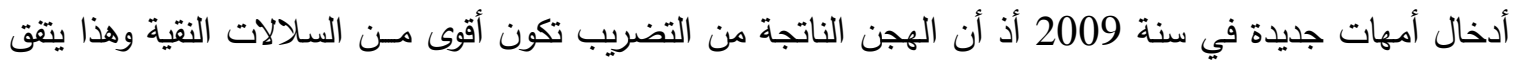
مع دراسة [18 ]عندما أجرى تضـريب بين الكارب الهنكاري والعراقي، ويتفق أيضاً مع ما قام به [19]عندما أجرى تضريب بين الكارب الاعتيادي والسمكة الذهبية في كندا فكانت الهجن الناتجة أقوى من السلالات النقية وتتفق هذه الدراسة مع مـا أشار اليه [20] أذ أظهرت النتائج أن الأجيال الناتجة من تضريب الأنثى العراقية مع الذكر الهنكـاري كانت متفوقة في صفة طول الجسم/الطول القياسي، ولم يجد [21 [أي فروقات معنوية في صفة عمق الجسم/ الطول

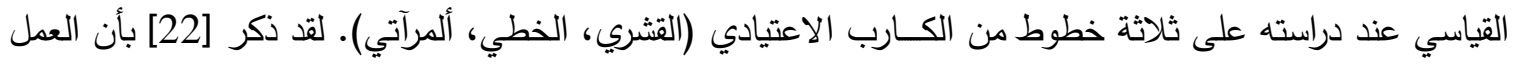
حول تحسين صفات الكـارب الاعتيادي المخصص للتربية أتجه نحو تجمع أكبر كمية من اللحم في الجسم من خلال تقليل حجم الرأس وزيادة عمق الجسم، فالكارب المخصص للتربية تكون نسبة عمق الجسم/الطول القياسي L من 0.3 - 0.5 بينما الكارب الموجود في الطبيعة (قبل التحسين) يتراوح مابين 0.25 - 0.30. وأجريت تجارب في فيتام لتضريب أسماك الكارب الهنكاري الذي يتميز بسرعة النمو لكن النضج الجنسي فيه متأخر والسمكة سريعة 
الأصابة بالأمراض مـع أسماك الكارب الفيتنامي الأبيض بطيء النمو وسريع النضج الجنسي والمقاوم للأمراض والمرغوب للاستهلاك المحلي أذ نجح الباحثون في أنتاج هجن تتميز بالنمو السريع ونسبة البقاء فيها مرتفعة [23]، وبين [24 ] في تجربة قام بها في هنكاريا والتي استمرت 12 شهرا أن الهجن الناتجة من تضريب أسماك الكارب الاعتيادي كانت متفوقة على متوسطة قيمة الإباء، إذ زاد متوسط النمو للهجن بنسبة 30\% في الصفات الجسمية والوزنية، وأوضح [25] أن تضريب خطوط مختلفة من الكارب الاعتيادي أدت إلى أعطاء نتائج جيدة في الزيادة الوزنية مما انعكست على قيم الصفات الجسمية للأسماك مثل الطول الكلي والطول القياسي وعمق الجسم. وبين [26] أنه يمكن الحصول على أبناء هجينة تمتلك بعض من الصفات أفضل من الأبناء النقية. وهذا يتفق مع [27]

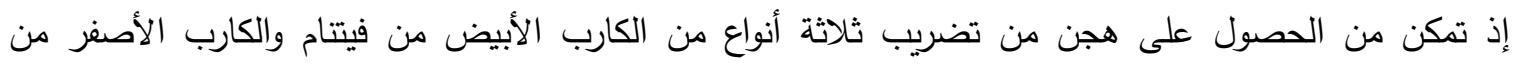

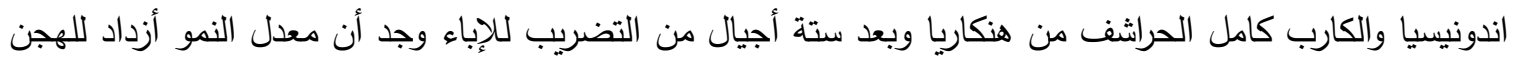
حوالي 33\% بالمقارنة مع الأسماك المستزرعة، وهذا ماأوصى به [28] من خلال دراسته من أنه يجب بصورة عملية على كيفية انتخاب أمهات لغرض تكون جيل جديد. ولأهمية الصفات الأنتاجية التكاثرية في مفاقس الأسماك بكاتك

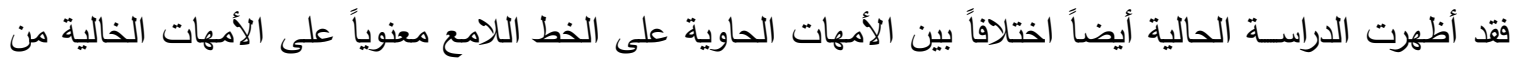
الخط اللامع في وزن البيض الكلي/غم وعدد البيض/كغم سمك وعدد البيض/غم بيض ووزن البيض /كغم سمك ونسبة الإخصاب وقطر البيضة، وقد يعود ذلك إلى أسباب عديدة مثل الاختلافات الوراثية بين الأمهات السلالة والعمر والعوامل البيئية ودرجة النضج الجنسي والحالة الفسيولوجية لأسماك التجربة، وكذلك قد يكون بسبب الأجيال الناتجة من خلال التضريب بعد أدخال قطيع من الأمهات سنة 2009 التي أدت إلى تخفيف أثار التربية الداخليـة التي عانت منها كثيراً الأسماك المستوردة عام 1982 [9]. وهذه النتائج تتناسب مع ماسجله [18] وكذللك جاءت متماثلة مع ما أشار اليه [9]، واختلفت مع ماسجله في دراسته [25] في بعض الصفات الأنتاجية التي انخفضت في الجيل الثالث للأكهات 2009 وقد عزا ذلك إلى صغر حجم الأمهات وعمرها أو بسبب الاختلافات بين الخطوط الوراثية والسلالة والتأقلم مع العوامل البيئية لكل جيل. ونستنتج من هذه الدراسة بأجراء دراسات أضافية معدقة (دراسة الـ DNA) من أجل التأكد من نسبة التداخل في الأسماك الجديدة مع الأسماك القديمة وتأثيرها على الصفات الأنتاجية من أجل اختيار الأفضل في المفاقس.

\section{CONFLICT OF INTERESTS.}

There are non-conflicts of interest .

1) المنظمة العربية للتتمية الزراعية (2008). دراسة حول تطوير تقانات الاستزراع السكي في الوطن العربي : 1 الصفحة.

2-Ockokwu, I.J.; Onyia, L.U.; and Ajijola, K.O.(2014). Effect of azanza garckeana (Goron tula) pulp meal inclusion on growth performance of Clarias gariepinus brood stock (burchell,1822). Nigeria Journal of Tropical Agriculture., 14: 134 -146.

3- Conn , E. \& Stumpf , P.K.(1976). Outline of Biochemistry. $4^{\text {th }}$ ed. John Wiley \&Sones, inc, New York, Colehester. Toronto \& Singapore : 629 pp. 
4) FAO. (2008). Fisheries and Aquaculture Development, culture Aquatic Species in formation programmers Cyprinus carpio.food and agriculture organization of the united nations. Rome : $14 \mathrm{p}$.

$$
\begin{aligned}
& \text { 5- جاسم , عبدالامير رحيم (2007). الطفيليات المصاحبة للأسماك المربات في ثلاثة محطات في محافظة } \\
& \text { البصرة، العراق. رسالة ماجستير , كلية الزراعة , جامعة البصرة : } 95 \text { صفحة. } \\
& \text { 6- السعدي، علي حمود (1994). استسال وتوصيف جين النمو في أسماك البز وإنتاج هرمونه في نظام بكتيري } \\
& \text { معبر ، رسالة دكتوراه ،كلية العلوم، جامعة بغداد. } \\
& \text { 7- العزاوي، احمد جاسم محمد.2004. دراسة بيئة العوالق النباتية في مبازل الجزء الثمالي للمصب العام. رسالة } \\
& \text { ماجستير ، كلية العلوم، جامعة بغداد. } \\
& \text { 8- الكبيسي، عبدالرحمن عبدالجبار • 1996. الواقع البيئي للمصب العام أطروحة دكتوراه، كلية التربية أبن الهيثث، } \\
& \text { جامعة بغداد. } 143 \text { صفحة. } \\
& \text { 9- نايف، طالب شمران (2005). بعض الصفات الأنتاجية التكاثرية لقطعان مفاقس الأسماك في محافظة بابل. }
\end{aligned}
$$

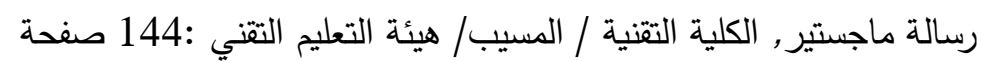

10- Gjedrem, T. (2005). Selection and Breeding Programs in Aquaculture, Springer ISBN- 10 1-4020-9. 364P.

11- Andrew, B.M. (2009).Review of Aquaculture Genetics and Genomics. Genomics and Reproduction Research Group. Institute of Aquaculture University of Stirling FK 4LA. Scotland UK.

12- صالح، خليل أبراهيم (2015). التكثير الاصطناعي للأسماك وإدارة المفاقيس. الكلية التقنية/ المسيب، منشورات

$$
\text { جامعة الفرات الأوسط التقنية، } 225 \text { صفحة. }
$$

13- كاظم , رائد عباس (2003). التحري عن الطفيليات القشرية على بعض أنواع الأسماك مزرعة أسماك الفرات,

مبزل المحاويل المجمع في محافظة بابل. رسالة ماجستير , كلية العلوم , جامعة بابل: 103 صفحة.

$$
\text { 14- احمد، هاشم عبدالرزاق (1991). مبادئ علم الأسماك. مطبعة جامعة البصرة : } 302 \text { صفحة. }
$$

15- Woynarovich, E. and Horvath , L.(1980). The artificial propagation of warm water finfish - Amanual for extension. F. O. W fishermen technical paper No. 201 , FiR / T201/ En/pp. 1 - 182

16- Alkunhi, K. H. Sukumaran, K. K. and Parameswaran, S.(1962). Induced spawing of the Chinese grass carp ctenopharyngodon idellas (C. and V.), and Silver carp, Hypophthalmie hthysmolitrix (C. and V.), in ponds at Cattaek, India. Tech. Pop.IpFc, (16): $22 \mathrm{P}$.

17- Duncan , D.B.(1955). Multiple range and multiple F test. Biometrics. 11: 1 - 42 p. 
18- الجبوري, معند عبيس عبدالله (2011). تتييم بعض الصفات الإنتاجية والتكاثرية من تضريب خطين مختلفين من أسماك الكارب الثائع (Cyprinus carpio L.). التقني :94 صفحة.

19- Taylor, J.and Mahon, R., 1977. Hybridization of Cyprinus carpio and Carassius auratus, the first two exotic species in the lower Laurentian Great Lakes. Env. Biol. Fish., 2: 205 -208.

20 - الجبوري, تيماء عبدالحسين مهدي كاظم (2012). استخدام المؤشرات الوراثية (Genetic Markers) في (لمائ

تقييم تضريب خطوط مختلفة من أسماك الكارب الثائع (Cyprinus carpio L.

$$
\text { التقنية / المسيب/ هيئة التعليم التقني :94 صفحة. }
$$

21- Alsaeedi, Nawar Khaled Teref.(2017). Phenotypic and Genotypic Analysis Among Different Common Carp (Cyprinus carpio) Population ,MSc. Thesis , Faculty of Agriculture (Saba Basha) / Alexandria University:75p.

22- Huet M. (1979). Text book of Fish culture, breeding cultivation of fish.Fishing news, Ltd, London. Pp: 114.

23- Austin , C.M.; Tuan ,P.A.; Binh , T.T.; Hung , L.Q. and Tan, N. T. (2007). Fish breeding practices And stock improvement strategies in Vietnam In relation to common carp. Research Institute for Aquaculture. 1, $119 \mathrm{Pp}$.

24- Uraiwan, S; Sumanojitraporn, S., Kamonrat W. and Keawrit, P.(2000).Effect of intrahybridization and introductory cross On growth and body shape of common carp , Cyprinus carpio (L.)

25- الصفار، أمير علاء هادي (2012). تتييم تتمية صغار أسماك الكارب الاعتيادي Cyprinus carpio

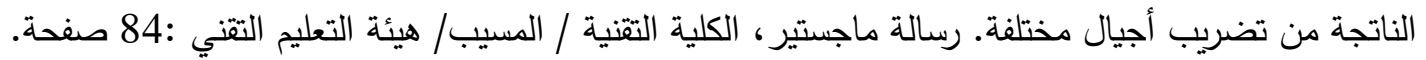

26- Zhou, J; Wu, Q.; Wang, Z. and Ye, Y. (2004)a. Genetic variation within and among six varieties of common carp (Cyprinus carpio L.) in China using microsatellite markers. Russian Journal of Genetics, 40 : 1144- 1148. doi: 10.1023/ B:RUGE.0000044758.51875.25.

27- Thien, T.M.(1996). Carp breeding in Vietnam Final Report submitted to IFS,15 P. 28- الحلي, عمار مضر سليمان (2009). تحديد التباين الوراثي والمظهري لأسماك الكارب الاعتيادي (Cyprinus carpio) 